upon-Tyne NHS Foundation Trust, comparing the 12 months directly preceding the pandemic (YR1) to the first 12 months of it (YR2). Variables included; presentation, etiology, stage, treatment and SARS-CoV-2 infection.

Results New referrals with hepatocellular carcinoma (HCC) fell for the 1st time in 15 years in YR2 $(35 \% ; 125$ in YR2 from 190 in YR1; Fig1A), with more presenting symptomatically. This was particularly striking for NAFLD patients (surveillance/incidental/symptomatic $32 / 55 / 13 \%$ in YR1 compared to $26 / 41 / 33 \%$ in YR2, $p=0.028$, Fig1B). Tumour size was greater in YR2 (med 44 vs $37 \mathrm{~mm}$; $\mathrm{p}=0.0165$, Fig1C), with 6 patients experiencing spontaneous hemorrhage in YR2. Despite challenges in YR2, patients received treatments appropriate for stage, in accordance with NHS waiting times. 11/ 125 (9\%) acquired SARS-CoV-2. None of these patients died due to their SARS-CoV-2 infection. Cases presenting with intrahepatic cholangiocarcinoma were also reduced in YR2, but less so (64 vs 76), with the commonest mode of presentation unchanged (symptomatic $\sim 75 \%$ ).

Conclusion The tragic consequences of the SARS-CoV-2 pandemic are evident for patients in our region - whose cancers are now more commonly presenting symptomatically. In our series, 9\% (11/125) of patients contracted SARS-CoV-2, of whom 3 subsequently died of advanced disease rather than SARS-CoV-2. At least $35 \%$ of our anticipated patients with HCC are 'missing'. If routine activities, including HCC surveillance, are not re-instituted and patients encouraged to attend, it is likely that they too will present with symptomatic advanced HCC.

\section{PWE-44 RESOURCE UTILISATION IN VARICEAL SURVEILLANCE PROGRAM DELIVERED WITHIN A NURSE-LED STABLE CIRRHOSIS CLINIC}

Muhammad Saleem*, Mohamed Elnagar, Alison Beard, Samantha Whyld, Andrew Austin. Royal Derby Hospital, Derby, UK

\subsection{6/gutjnl-2021-BSG.234}

Introduction Bleeding from GOV represents 30\% of total acute upper GI haemorrhage with mortality of up to $20 \%$ at initial presentation. Whilst mortality in Child A disease should be $<10 \%$, each emergency presentation is expensive. The GIRFT report recommends developing a database for cirrhosis in order to ensure a systematic approach to varices surveillance, something we have delivered via a Nurse led stable cirrhosis clinic since 2016. While BSG guidelines recommended screening for all patients with cirrhosis or PHT, we have adopted Baveno VI consensus workshop criteria $(\mathrm{TE}<20 \mathrm{kPa}$ or platelet count $>150$ ) to safely avoid gastroscopy in this cohort. Grade2/3 varices required primary prophylaxis with banding (VBL) or non-selective beta blockers (NSBB). In 2016, prophylaxis for grade 1 varices was not recommended.

Methods We conducted a retrospective review for all the cirrhotic patients seen in our nurse-led stable cirrhosis clinic during the first year of its establishment

Results In 2016, a total of 53(40 male) cirrhotic patients were reviewed in the clinic (ALD 25, NASH 9, other 20). Other aetiologies included HCV, PBC and HFE-related cirrhosis. Only 3 patients had Child Pugh B with the rest having Child A disease. No patient had ever suffered variceal bleeding. 26/ 53 (49\%) patients met the exclusion criteria set by Baveno VI consensus and would have avoided OGD. 12/26 had their index OGD which had been ordered prior to the instigation of the nurse-led clinic and reassuringly none showed varices. Screening was indicated for the remaining 27 patients. One patient passed away before his surveillance appointment. Grade I OV were diagnosed in 13/27 (48\%) patients, of whom 7 inappropriately received NSBB and the remainder had follow up OGD at one year. Grade II varices were identified in $2 / 27(7.5 \%)$ and band ligation performed. In the remaining $11 / 27$ no varices were found and with annual improvements in fibroscan measurements only 5/11 required follow up OGD. None of the patients had variceal bleed in follow up 5 year period.

Conclusions Variceal screening is an important step in identifying high risk varices. By following Baveno VI criteria in this cohort, we have conformed the potential to reduce demand for OGD by around 50\% for immediate OGD and another $50 \%$ of the remainder whose fibroscan results improve with abstinence from alcohol without subsequent bleeding events. Our review also shows an opportunity to improve resource utilisation by refreshing adherence to the existing guidelines.

\section{PWE-45 BACTERAEMIA, SEPSIS, AND ANTIBIOTIC RESISTANCE IN AUSTRALIAN PATIENTS WITH CIRRHOSIS: A POPULATION-BASED STUDY}

\begin{abstract}
${ }^{1,2}$ Amy Johnson*, ${ }^{3}$ Isanka Ratnasekera, ${ }^{3}$ Katharine Irvine, ${ }^{4,5}$ Andrew Henderson, ${ }^{1,2}$ Elizabeth Powell, ${ }^{6}$ Patricia Valery. ${ }^{1}$ Princess Alexandra Hospital, Department of Gastroenterology and Hepatology, Brisbane, Australia; ${ }^{2}$ Centre for Liver Disease Research, Faculty of Medicine, The University of Queensland, Brisbane, Australia; ${ }^{3}$ Mater Research Institute, Translational Research Institute, The University of Queensland, Brisbane, Australia; ${ }^{4}$ Infection Management Services, Princess Alexandra Hospital, Brisbane, Australia; ${ }^{5}$ University of Queensland Centre for Clinical Research, Herston, Brisbane, Australia; ${ }^{6}$ QIMR Berghofer Medical Research Institute, Brisbane, Australia
\end{abstract}

\subsection{6/gutjnl-2021-BSG.235}

Introduction Multiple factors predispose patients with cirrhosis to sepsis and/or bacteraemia and this has a high mortality rate. Within different geographic regions there are marked differences in the prevalence of infection with multidrug resistant organisms (MDRO). This study examined risk factors for and outcomes of sepsis/bacteraemia in Australian public hospital admissions with cirrhosis over the last decade, along with the bacterial pathogens responsible and their antibiotic susceptibility profiles.

Methods A population-based retrospective cohort study was conducted from 1 January 2008 to 31 December 2017. Hospital admissions for patients with a diagnosis of cirrhosis were categorized by the presence or absence of sepsis/bacteraemia. Clinical and socio-demographic information including cirrhosis aetiology, complications and comorbidities, and in-hospital mortality were examined using bivariate and multivariate analyses. In patients with bacteraemia, the type and prevalence of bacteria and antibiotic resistance was assessed.

Results Sepsis/bacteraemia was present in 3,951 of 103,165 hospital admissions with a diagnosis of cirrhosis. Factors associated with sepsis/bacteraemia included disease aetiology, particularly primary sclerosing cholangitis (adj-OR $=15.09,95 \% \mathrm{CI}$ 12.24-18.60), alcohol (adj-OR $=2.90,95 \% \mathrm{CI}$ 2.71-3.09), Charlson co-morbidity index $\geq 3$ (adj-OR=3.54, 95\%CI 3.193.93), and diabetes (adj-OR $=1.87$, 95\%CI 1.74-2.01). Overall case-fatality rate among admissions with sepsis/bacteraemia was $27.7 \%$ (95\%CI, 26.3\%-29.1\%) vs $3.0 \%$ (95\%CI, $28.9 \%$ $31.1 \%$ ) without sepsis/bacteraemia. Patients with sepsis/ 
bacteraemia were 6.5 times more likely to die in hospital than to be discharged alive (adj-OR=6.50, 95\%CI 5.95-7.11). The most common organisms identified were Escherichia coli and Staphylococcus aureus, present in $22.9 \%$ and $18.1 \%$ respectively, of the 2,265 admissions with a positive blood culture. The prevalence of MDR bacteria was low (5.6\%).

Conclusions Morbidity and mortality related to sepsis/bacteraemia in patients with cirrhosis remains a critical clinical problem.

\section{PWE-46 IMPACT OF THE COVID-19 PANDEMIC ON PATIENTS WITH HEPATOCELLULAR CARCINOMA IN THE WEST OF SCOTLAND}

${ }^{1}$ Alistair Mclaren*, ${ }^{2}$ Johannes Spoor, ${ }^{1}$ Gregory Naylor, ${ }^{1}$ Douglas Cartwright, ${ }^{2}$ Adrian J Stanley, 'Thomas Ronald Jeffrey Evans. 'Beatson West of Scotland Cancer Centre, Glasgow, UK; ${ }^{2}$ Glasgow Royal Infirmary, Glasgow, UK

\subsection{6/gutjnl-2021-BSG.236}

Introduction The COVID-19 pandemic has focused medical resources on acute and critical care, with an impact on patients with chronic illness. Worldwide, HCC surveillance, imaging and clinic follow-up may have been impacted, which is likely to worsen outcomes. In the West of Scotland, all patients with HCC are discussed at our weekly regional MDT. We examined the difference in outcomes for new patients referred to this MDT and patients returning to the MDT to see if there were differences during the COVID-19 pandemic compared to the previous year.

Methods We collected data on patients reviewed in the West of Scotland HCC MDT between April - October 2020; compared to the same period in 2019. Statistical analysis was performed using ' $\mathrm{R}$ ' ( $\mathrm{R}$ Foundation for Statistical Computing, Vienna, Austria).

Results There were 128 patients discussed in April - October 2020: 69 patients presenting to the MDT for the first time ('new') and 59 patients that had been discussed previously ('return') compared to 155 (93 new) in April - October 2019. Amongst new patients discussed, median overall survival was not reached, but 12-month survival rate was 30\% (95\% CI $0.13-0.70)$ in 2020 compared to $54 \%$ in 2019 (95\%CI $0.44-0.64, p=0.10)$. For return and new patients combined, the median overall survival was 11.3 months in 2020 (95\% CI 6.93 - not reached) vs 16.9 months in 2019 (95\% CI 13.8 - not reached), and 1 year survival rate was $48 \%$ in 2020 (95\% CI $0.38-0.62$ ) compared to $62 \%$ in 2019 (95\%CI $0.55-0.70)$. As seen in figure 1 , there was a significant decrease in survival in combined new and return patients with HCC presented to the MDT in 2020 compared to 2019 ( $p=$ 0.04) There was no significant difference in median Child Pugh score (A6 in both 2020 and 2019), median ECOG performance status (1 in both 2020 and 2019), median AFP $(12.0 \mathrm{kU} / \mathrm{L}$ in $2020,7.0 \mathrm{kU} / \mathrm{L}$ in $2019 p=0.28)$, and median diameter of largest lesion $(4.1 \mathrm{~cm}$ in $2020,3.0 \mathrm{~cm}$ in 2019 $p=0.17)$. A higher proportion of patients were referred for TACE (20\% vs 15\%), and Best Supportive Care (43\% vs $32 \%)$ in 2020 compared to 2019 , with a lower proportion being referred for RFA (11\% vs 15\%) and a similar proportion for SACT (11\%) and surgery (3\%).

Conclusions We identified significantly reduced survival in patients with HCC assessed at our MDT during the COVID19 pandemic compared with the same period in 2019. This is despite no significant difference in factors that influence survival. This may continue to have implications continuing during recovery from the pandemic and requires further study.

\section{PWE-47 CLINICAL PRESENTATION AND OUTCOMES OF WILSON'S DISEASE: A SINGLE CENTRE COHORT STUDY}

Akhilesh Mulay*, James Dooley, Emmanouil Tsochatzis. UCL Institute for Liver and Digestive Health, Royal Free Hospital and UCL, London, UK

\subsection{6/gutjnl-2021-BSG.237}

Introduction Wilson's Disease is a disorder of copper homeostasis leading to hepatic and neuropsychiatric disease. Clinical data on these patients are limited due to low disease frequency. This report explores the presentation, management and outcomes of a large cohort of patients with Wilson's disease including pregnancy outcomes.

Methods A retrospective analysis was performed on 63 patients with Wilson's disease managed at the Royal Free Hospital, London, between 2012 and 2021. Clinical data were collected on presenting features, investigations, treatment and outcomes.

Abstract PWE-46 Figure 1 Overall Survival of all patients with HCC discussed in the MDT in 2019 (Pre COVID) vs 2020 (During COVID) 